\title{
Association between the markers of metabolic acid load and higher all-cause and cardiovascular mortality in a general population with preserved renal function
}

\begin{abstract}
Minseon Park ${ }^{1}$, Sung Jae Jung ${ }^{2}$, Seoyoung Yoon ${ }^{2}$, Jae Moon Yun ${ }^{1}$ and Hyung-Jin Yoon ${ }^{2}$
Although metabolic acid load has been associated with many well-known risk factors for mortality, its clinical implications are not yet clear. To evaluate the association between biomarkers of metabolic acid load, such as serum bicarbonate, serum anion gap and urine $\mathrm{pH}$ and mortality, we analyzed the health records of 31590 adults who underwent a health screening between January 2001 and December 2010 and had an estimated glomerular filtration rate $\geqslant 60 \mathrm{ml} \mathrm{min}^{-1}$ per $1.73 \mathrm{~m}^{2}$. Urine pH was measured by a dipstick test performed on fast morning urine sample and categorized as acidic (urine $\mathrm{pH} \leqslant 5.5$ ), neutral and alkaline (urine $\mathrm{pH} \geqslant 8.0$ ). Using the Cox proportional hazard model, the adjusted hazard ratio (aHR) of all-cause mortality of the lowest quartile of serum bicarbonate was 1.460 (95\% confidence interval $(\mathrm{Cl})$ 1.068-1.995) compared with the highest quartile, after a median follow-up of 93 months. The aHRs of cardiovascular and cancer mortality of the lowest quartile of serum bicarbonate were 2.647 (95\% Cl 1.148-6.103) and 1.604 (95\% Cl 1.024-2.513), respectively, compared with the highest quartile. Acidic and neutral urine $\mathrm{pH}$ were significantly associated with a higher all-cause mortality (aHR $2.550,95 \% \mathrm{Cl}$ 1.316-4.935; aHR 2.376 95\% Cl 1.254-4.501, respectively), compared with an alkaline urine $\mathrm{pH}$. In conclusion, higher metabolic acid load was associated with an increased all-cause and cardiovascular mortality in a healthy population. The association between metabolic acid load and mortality and the causality of the relationship need to be confirmed.
\end{abstract}

Hypertension Research (2015) 38, 433-438; doi:10.1038/hr.2015.23; published online 12 March 2015

Keywords: all-cause mortality; cardiovascular mortality; metabolic acid load; serum bicarbonate; urine pH

\section{INTRODUCTION}

Metabolic acidosis is a common complication of moderate-to-severe chronic kidney disease (CKD) and primarily results from an inadequate excretion of the daily metabolic acid load. Metabolic acidosis has been associated with a poor renal outcome and higher mortality in patients with CKD. ${ }^{1}$ There are several reports suggesting that the clinical implications of metabolic acidosis may extend beyond the subjects with CKD and that clinical biomarkers of metabolic acidosis even within the normal range may be associated with clinical conditions, such as insulin resistance or higher levels of inflammatory biomarkers. ${ }^{2,3}$

In cross-sectional studies, the clinical indices of metabolic acidosis, such as lower serum bicarbonate, higher serum anion gap or acidic urine $\mathrm{pH}$, have been associated with well-known risk factors for premature mortality, such as insulin resistance, ${ }^{2}$ higher levels of inflammatory biomarkers, ${ }^{3}$ visceral obesity and metabolic syndrome, ${ }^{4}$ high blood pressure (BP), ${ }^{5,6}$ and lower cardiorespiratory fitness ${ }^{7}$ in the general population. In prospective studies of women, a higher plasma bicarbonate level was associated with lower odds of incident type 2 diabetes $^{8}$ and hypertension. ${ }^{9}$ Lower serum bicarbonate levels have been associated with the rapid decline of the estimated glomerular filtration rate (eGFR) or higher risk of incident eGFR $<60 \mathrm{ml} \mathrm{min}^{-1}$ per $1.73 \mathrm{~m}^{2}$ in community living subjects with a relatively normal renal function. ${ }^{10,11}$

Despite several studies observing an association between well-known risk factors for mortality and metabolic acid load as represented by clinical biomarkers of metabolic acidosis, such as lower serum bicarbonate, higher serum anion gap or acidic urine $\mathrm{pH}$, irrespective of the presence of metabolic acidemia, direct evidence on the association between metabolic acid load and mortality in the general population with a preserved renal function is very limited. Recently, Raphael et al. ${ }^{12}$ reported that low serum bicarbonate levels were significantly associated with a higher mortality in people with CKD but not in people without CKD. Therefore, the long-term clinical implications of metabolic acid load in the general population are not clear yet.

An increase in daily metabolic acid load in the general population with a preserved renal function is believed to result from acidogenic modern diets that differs from ancestral hominid diets in many aspects including acid-base balance. ${ }^{13,14}$ It is important to evaluate the

${ }^{1}$ Department of Family Medicine, Seoul National University Hospital, Seoul, Republic of Korea and ${ }^{2}$ Department of Biomedical Engineering, Seoul National University College of Medicine, Seoul, Republic of Korea

Correspondence: Professor H-J Yoon, Department of Biomedical Engineering, Seoul National University College of Medicine, 101 Daehak-ro, Jongno-gu, Seoul 110-744, Republic of Korea. E-mail hjyoon@snu.ac.kr

Received 19 October 2014; revised 29 December 2014; accepted 25 January 2015; published online 12 March 2015 
connection between metabolic acid load and mortality to develop a strategy to prevent any premature mortality through diet modification or bicarbonate replacement.

To evaluate the association between metabolic acid load and mortality, we examined the health screening data of 31590 apparently healthy adults with eGFR $60 \mathrm{ml} \mathrm{min}^{-1}$ per $1.73 \mathrm{~m}^{2}$ or higher.

\section{METHODS}

\section{Participants and data collection}

Between January 2001 and December 2010, a total of 61033 health screenings were performed at the Health Promotion Centre of Seoul National University Hospital. We excluded 20693 repeated screenings and 3908 screenings with missing data on serum bicarbonate or creatinine. After further exclusion of 4842 screenings of participants younger than 20 years of age, eGFR value $<60 \mathrm{ml} \mathrm{min}^{-1}$ per $1.73 \mathrm{~m}^{2}$, or survival duration of 24 months or less after the baseline health screening, we analyzed the health records of the first screening visits of 31590 adults. The study protocol was approved by the Institutional Review Board of the Seoul National University Hospital.

Information on the history of smoking, alcohol ingestion, regular exercise, diabetes, hypertension and pharmacological treatment for diabetes and/or hypertension was obtained by using a structured, self-reported questionnaire and validated by direct interview with trained nurses. Trained physicians interviewed and examined all participants just before the health screening. The smoking status was determined into the following three categories: current smokers, ex-smokers and non-smokers. Participants who smoked at least one cigarette per day at the time of the health screening were classified as current smokers and those who reported that they did not smoke at the time of the health screening but had previously smoked were classified as ex-smokers. Participants who ingested alcoholic beverages at least once a week were classified as regular drinkers. Regular exercise was defined as exercise longer than $30 \mathrm{~min}$ at least three times per week.

Height and weight were measured after an overnight fast and after voiding urine. Body mass index (BMI) was calculated by dividing the weight in kilograms by squared height in meters. BP was measured using an automated BP measurement device (Jawon, Busan, Korea) after resting for at least 20 min in a sitting position.

Blood samples were drawn after a 12-h overnight fast. All the biochemical tests including serum bicarbonate were performed with venous blood samples. Serum creatinine was measured by using the Jaffe alkaline picrate method with a Toshiba 200FR (Toshiba Medical Systems, Tokyo, Japan). The serum creatinine measurement was reduced by $5 \%$ to standardize to isotope dilution mass spectrometry as previously suggested. ${ }^{15}$ eGFR was calculated with the Chronic Kidney Disease Epidemiology Collaboration creatinine equation. ${ }^{16}$ Serum biochemical markers including bicarbonate were measured using the enzymatic method with a Toshiba 200FR (Toshiba Medical Systems, Tokyo, Japan). The normal range of the bicarbonate of the venous serum in Seoul National University Hospital was $24-31 \mathrm{mEql}^{-1}$. The serum anion gap was calculated as the serum sodium level $\left(\mathrm{mEql}^{-1}\right)$ minus the serum chloride $\left(\mathrm{mEql}^{-1}\right)$ and serum bicarbonate level $\left(\mathrm{mEql}^{-1}\right)$. The serum albuminadjusted anion gap was calculated as previously proposed. ${ }^{7}$

Urine $\mathrm{pH}$ and albuminuria were determined semi-quantitatively with singlespot urine dipstick analysis (YD Diagnostics, Yong-In, Korea) performed on morning urine samples after overnight fasting. Dipstick analysis for albuminuria was reported as negative, trace, $1+, 2+, 3+$ or $4+$ and albuminuria was defined as $1+$ or higher. Kraut and Madias ${ }^{17}$ have reviewed that in the absence of impaired renal acidification, urine $\mathrm{pH} 5.5$ or lower represented higher metabolic acid load. Acidic urine was defined as urine $\mathrm{pH} 5.5$ or lower, neutral urine as urine $\mathrm{pH}$ between 6.0 and 7.5 and alkaline urine as urine $\mathrm{pH} 8.0$ or higher. All tests were conducted by routine clinical processing in a clinical laboratory of Seoul National University Hospital, which was inspected and surveyed annually by the Korean Association of Quality Assurance for Clinical Laboratories.

\section{Outcome and follow-up}

The outcome of interest was all-cause and cause-specific mortality. The participants were followed up for mortality until 31 December 2012. Death was confirmed by death certificate data from the National Statistical Office of Korea by using the personal identification number assigned at birth. The cause of death was classified according to the International Classification of Disease, 10th revision.

\section{Statistical analysis}

Statistical analyses were conducted with SPSS IBM 19 (IBM, Chicago, IL, USA). The distribution of demographic and clinical parameters across the quartile groups of serum bicarbonate was compared with the analysis of variance for continuous variables and $\mathrm{X}^{2}$ test for discrete variables. Two-sided $P$-values $<0.05$ were considered statistically significant. The association between the clinical biomarkers of metabolic acid load such as quartile groups of serum bicarbonate and anion gap value and urine acidity (urine $\mathrm{pH} \leqslant 5.5,6.0-7.5$, $\geqslant 8.0$ ) and all-cause and cause-specific mortality was analyzed with the Cox proportional hazard model, adjusted for baseline variables such as age, sex, smoking status, regular alcohol ingestion, regular exercise, history of diabetes and/or hypertension medication, BMI, systolic BP, serum calcium, serum phosphorus, serum uric acid, fasting serum glucose, serum albumin, serum high-density lipoprotein-cholesterol (HDL-Chol), serum triglyceride, eGFR and albuminuria.

To evaluate the role of sex, obesity and serum uric acid in the association between metabolic acid load and mortality, subgroup analyses were performed according to sex, BMI $\left(<23 \mathrm{~kg} \mathrm{~m}^{-2}, \geqslant 23 \mathrm{~kg} \mathrm{~m}^{-2}\right)^{18}$ and the sex-specific median level of serum uric acid $\left(5.8 \mathrm{mg} \mathrm{dl}^{-1}\right.$ in men and $4.2 \mathrm{mg} \mathrm{dl}^{-1}$ in women). Tests for interaction were performed by introducing an interaction term into the adjusted Cox proportional hazard model.

\section{RESULTS}

Table 1 summarizes the general characteristics of the participants at baseline. The mean age of the participants was $49.9 \pm 10.7$ years and $50.9 \%$ of the participants were men. The mean age decreased with decreasing quartiles of serum bicarbonate. The proportion of participants who were currently smoking or not exercising regularly increased with decreasing quartiles of serum bicarbonate. The distribution of the participants who ingested alcohol regularly was not different across the quartile groups of serum bicarbonate. The proportion of participants with a history of taking anti-hypertensive medications decreased with decreasing quartiles of serum bicarbonate. The distribution of participants with a history of diabetic medications was a $U$ shape with the lowest number in the second quartile of serum bicarbonate. Although the distribution of albuminuria was not different across the serum bicarbonate quartile groups, the mean eGFR increased with decreasing quartiles of serum bicarbonate (Table 1).

The estimated mean serum bicarbonate levels were negatively associated with the serum anion gap after adjusted for baseline variables such as age, sex, smoking status, regular exercise, alcohol ingestion, history of medication for diabetes and/or hypertension, BMI, systolic BP, fasting serum glucose, serum calcium, serum phosphorus, serum uric acid, serum albumin, serum triglyceride, serum high-density lipoprotein-cholesterol, eGFR and albuminuria ( $\beta-0.641,95 \%$ confidence interval $(\mathrm{CI})-0.652,-0.632$; data not shown). The estimated mean serum bicarbonate levels were positively associated with urine $\mathrm{pH}$ after adjustment for baseline variables such as age, sex, smoking status, regular exercise, alcohol ingestion, history of medication for diabetes and/or hypertension, BMI, systolic BP, fasting serum glucose, serum calcium, serum phosphorus, serum uric acid, serum albumin, serum triglyceride, serum high-density lipoprotein-cholesterol, eGFR and albuminuria $\left(28.1 \mathrm{mEql}^{-1}\right.$ in acidic urine, $95 \%$ CI $27.9-28.2 \mathrm{mEql}^{-1} ; 28.9 \mathrm{mEql}^{-1}$ in neutral 
Table 1 General characteristics at health screening of the participants by serum bicarbonate quartiles ${ }^{\mathrm{a}}$

\begin{tabular}{|c|c|c|c|c|c|c|}
\hline Serum Bicarbonate $\left(m E q I^{-1}\right)$ & $1 Q(\leqslant 26)$ & $2 Q(27-28)$ & $3 Q(29-30)$ & $4 Q(\geqslant 31)$ & Total & P-value ${ }^{b}$ \\
\hline Number of Participants & 6134 & 8110 & 8968 & 8378 & 31590 & \\
\hline Men (\%) & $46.8 \%$ & $49.1 \%$ & $51.2 \%$ & $55.3 \%$ & $50.9 \%$ & $<0.001$ \\
\hline Age at screening (years) & $48.0 \pm 11.0$ & $49.1 \pm 10.9$ & $50.2 \pm 10.6$ & $51.7 \pm 10.1$ & $49.9 \pm 10.7$ & $<0.001$ \\
\hline \multicolumn{7}{|l|}{ Smoking } \\
\hline Non-smoker (\%) & $54.4 \%$ & $55.9 \%$ & $55.6 \%$ & $54.4 \%$ & $55.1 \%$ & \multirow[t]{3}{*}{$<0.001$} \\
\hline Ex-smoker (\%) & $18.9 \%$ & $20.5 \%$ & $22.0 \%$ & $24.8 \%$ & $21.8 \%$ & \\
\hline Current smoker (\%) & $26.8 \%$ & $23.6 \%$ & $22.4 \%$ & $20.8 \%$ & $23.1 \%$ & \\
\hline Regular exercise (\%) & $37.1 \%$ & $39.0 \%$ & $40.0 \%$ & $42.7 \%$ & $39.9 \%$ & $<0.001$ \\
\hline Regular alcohol intake (\%) & $49.6 \%$ & $50.7 \%$ & $51.0 \%$ & $50.6 \%$ & $50.6 \%$ & 0.400 \\
\hline History of anti-HT medication (\%) & $11.8 \%$ & $12.6 \%$ & $13.0 \%$ & $15.6 \%$ & $13.4 \%$ & $<0.001$ \\
\hline History of DM medication (\%) & $6.4 \%$ & $6.1 \%$ & $6.2 \%$ & $7.7 \%$ & $6.6 \%$ & $<0.001$ \\
\hline Systolic blood pressure (mmHg) & $130.4 \pm 18.3$ & $130.2 \pm 18.5$ & $130.3 \pm 18.4$ & $130.9 \pm 18.1$ & $130.5 \pm 18.3$ & 0.067 \\
\hline Diastolic blood pressure $(\mathrm{mmHg})$ & $77.8 \pm 12.0$ & $77.8 \pm 12.1$ & $77.9 \pm 11.9$ & $78.2 \pm 11.7$ & $77.9 \pm 11.9$ & 0.096 \\
\hline Body mass index $\left(\mathrm{kg} \mathrm{m}^{-2}\right)$ & $24.2 \pm 3.3$ & $24.0 \pm 3.1$ & $23.9 \pm 3.0$ & $23.7 \pm 2.9$ & $24.0 \pm 3.1$ & $<0.001$ \\
\hline Body mass index $\geqslant 23 \mathrm{~kg} \mathrm{~m}^{-2}(\%)^{c}$ & $3859 / 6130(63.0 \%)$ & $4996 / 8110(61.6 \%)$ & $5454 / 8965(60.8 \%)$ & $4943 / 8377$ (59.0\%) & $19252 / 31582$ (61.0\%) & $<0.001$ \\
\hline Serum calcium (mg dl-1) & $9.25 \pm 0.39$ & $9.26 \pm 0.39$ & $9.29 \pm 0.37$ & $9.35 \pm 0.37$ & $9.29 \pm 0.38$ & $<0.001$ \\
\hline Serum phosphorus (mg dl-1) & $3.46 \pm 0.53$ & $3.49 \pm 0.52$ & $3.52 \pm 0.52$ & $3.54 \pm 0.52$ & $3.51 \pm 0.52$ & $<0.001$ \\
\hline Serum albumin $\left(\mathrm{g} \mathrm{dl}^{-1}\right)$ & $4.35 \pm 0.26$ & $4.36 \pm 0.26$ & $4.39 \pm 0.26$ & $4.42 \pm 0.25$ & $4.38 \pm 0.26$ & $<0.001$ \\
\hline Serum uric acid $\left(\mathrm{mg} \mathrm{dl^{-1 } )}\right.$ & $5.04 \pm 1.41$ & $5.04 \pm 1.34$ & $5.08 \pm 1.31$ & $5.14 \pm 1.28$ & $5.08 \pm 1.33$ & $<0.001$ \\
\hline Fasting serum glucose $\left(\mathrm{mg} \mathrm{dl}^{-1}\right)$ & $97.9 \pm 24.8$ & $96.9 \pm 23.0$ & $96.9 \pm 22.7$ & $97.2 \pm 22.2$ & $97.2 \pm 23.1$ & 0.042 \\
\hline Serum cholesterol (mg dl-1) & $196.2 \pm 35.6$ & $197.8 \pm 34.8$ & $201.6 \pm 35.1$ & $203.5 \pm 35.0$ & $200.1 \pm 35.2$ & $<0.001$ \\
\hline Serum triglyceride $\left(\mathrm{mg} \mathrm{dl}^{-1}\right)$ & $135.3 \pm 95.7$ & $132.2 \pm 89.5$ & $133.6 \pm 89.3$ & $133.3 \pm 83.3$ & $133.5 \pm 89.1$ & 0.225 \\
\hline Serum HDL-Cholesterol (mg dl-1) & $55.2 \pm 18.2$ & $55.3 \pm 18.4$ & $56.1 \pm 18.6$ & $57.3 \pm 20.2$ & $56.0 \pm 18.9$ & $<0.001$ \\
\hline Serum Creatinine $\left(\mathrm{mg} \mathrm{dl}^{-1}\right)$ & $0.96 \pm 0.15$ & $0.96 \pm 0.15$ & $0.97 \pm 0.15$ & $0.99 \pm 0.15$ & $0.97 \pm 0.15$ & $<0.001$ \\
\hline 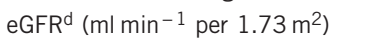 & $82.3 \pm 12.7$ & $81.9 \pm 12.2$ & $80.8 \pm 11.6$ & $79.0 \pm 11.0$ & $80.0 \pm 11.9$ & $<0.001$ \\
\hline Albuminuria $(\%)$ & $6.9 \%$ & $6.6 \%$ & $6.9 \%$ & $6.4 \%$ & $6.7 \%$ & 0.550 \\
\hline Serum anion gap $\left(\mathrm{mEq} \mathrm{I}^{-1}\right)$ & $10.7 \pm 2.9$ & $9.0 \pm 2.4$ & $8.0 \pm 2.3$ & $6.7 \pm 2.3$ & $8.4 \pm 2.8$ & $<0.001$ \\
\hline \multicolumn{6}{|l|}{ Urine $\mathrm{pH}(\%)^{\mathrm{f}}$} & \multirow[t]{4}{*}{$<0.001$} \\
\hline$\leqslant 5.5$ & 1988/5874 (33.8\%) & 2169/7781 (27.9\%) & $1905 / 8684(21.9 \%)$ & 1199/8220 (14.6\%) & $7261 / 30559$ (23.8\%) & \\
\hline $6.0-7.5$ & $3419 / 5874(58.2 \%)$ & $4747 / 7781(61.0 \%)$ & $5361 / 8684(61.7 \%)$ & $5239 / 8220(63.7 \%)$ & $18766 / 30559$ (61.4\%) & \\
\hline$\geqslant 8.0$ & $467 / 5874$ (8.0\%) & 865/7781 (11.1\%) & $1418 / 8684(16.3 \%)$ & $1782 / 8220(21.7 \%)$ & $4532 / 30559$ (14.8\%) & \\
\hline
\end{tabular}

Abbreviations: ANOVA, analysis of variance; DM, diabetes; eGFR, estimated glomerular filtration rate; HDL, high-density lipoprotein; HT, hypertension.

aData represent mean \pm s.d. or proportion.

bBased on ANOVA for continuous variables and $\mathrm{X}^{2}$-test for discrete variables.

${ }^{\mathrm{C}}$ Missing in eight participants.

Calculated with the Chronic Kidney Disease Epidemiology Collaboration creatinine equation.

eSpot urine dipstick test for albuminuria 1+ or higher.

${ }^{\mathrm{f}}$ Missing in 1031 participants.

urine, 95\% CI 28.8-29.0 $\mathrm{mEq}^{-1} ; 29.8 \mathrm{mEq}^{-1}$ in alkaline urine, 95\% CI 29.7-29.9 $\mathrm{mEq}^{-1}$; $P$ for trend $<0.001$; data not shown).

During a median follow-up of 93 months, 398 deaths, including 213 cancer deaths and 53 cardiovascular deaths, were observed. With adjustment for baseline variables such as age, sex, smoking status, regular exercise, alcohol ingestion, history of medication for diabetes and/or hypertension, BMI, systolic BP, fasting serum glucose, serum calcium, serum phosphorus, serum uric acid, serum albumin, serum triglyceride, serum high-density lipoprotein-cholesterol, eGFR and albuminuria, the lowest quartile of serum bicarbonate was significantly associated with an increased risk of all-cause mortality compared to the highest quartile (adjusted hazard ratio (aHR) 1.460, 95\% CI 1.068-1.995; Table 2). The aHRs of cardiovascular and cancer mortality in the participants in the lowest quartile of serum bicarbonate were significantly higher compared with those in the highest quartile (aHR 2.647, 95\% CI 1.148-6.103; 1.604, 1.024-2.513, respectively; Table 2). Mortality because of causes other than cardiovascular disease and cancer was not significantly different across the serum bicarbonate quartile groups. After exclusion of 428 participants who were diagnosed with chronic obstructive pulmonary disease (COPD) within 12 months after the health screening, the level of the association did not change (aHR for all-cause mortality in the lowest quartile of serum bicarbonate 1.439, 95\% CI 1.050-1.973, compared to the highest quartile; data not shown). After exclusion of participants with a history of taking diabetic and/or anti-hypertensive medication, the risk of total death for each quartile group of serum bicarbonate was elevated slightly (aHR 1.797, 95\% CI 1.224-2.639 in the lowest quartile group compared to the highest quartile group; data not shown).

With subgroup analysis, the association between the quartile groups of serum bicarbonate and all-cause mortality was not significantly different according to sex (aHR 1.297, 95\% CI 0.889-1.893 in men in the lowest quartile compared to those in the highest quartile; aHR $1.671,95 \%$ CI $0.947-2.948$ in women in the lowest quartile compared to those in the highest quartile; $\mathrm{p}$ for interaction 0.786). With subgroup analysis according to BMI, the risk for all-cause mortality in the lowest quartile of serum bicarbonate compared to the highest quartile significantly increased only in participants with BMI higher than $23 \mathrm{~kg} \mathrm{~m}^{-2}$ (aHR 1.184, 95\% CI 0.711-1.973 in participants with BMI equal to $23 \mathrm{~kg} \mathrm{~m}^{-2}$ or lower; aHR 1.601, 95\% CI 1.071-2.393 in participants with BMI higher than $23 \mathrm{~kg} \mathrm{~m}^{-2}$; $\mathrm{p}$ for interaction 0.743 ; Table 3). With subgroup analysis according to the sex-specific median 
Table 2 The association between serum bicarbonate level and all-cause, cardiovascular and cancer mortality

\begin{tabular}{|c|c|c|c|c|c|}
\hline Causes of death (person-year) & $1 Q(48600)$ & $2 Q(61677)$ & $3 Q(65619)$ & $4 Q(60376)$ & Total (236 274) \\
\hline \multicolumn{6}{|l|}{ All-cause } \\
\hline Number of deaths & 107 & 98 & 98 & 95 & 398 \\
\hline Incidence density ${ }^{a}$ & 220.2 & 158.9 & 149.3 & 157.3 & 168.4 \\
\hline $\mathrm{aHR}$ & 1.460 & 1.119 & 1.095 & Reference & - \\
\hline$(95 \% \mathrm{Cl})^{\mathrm{b}}$ & (1.068-1.995) & $(0.819-1.530)$ & $(0.804-1.493)$ & & \\
\hline \multicolumn{6}{|l|}{ Cardiovascular } \\
\hline Number of deaths & 19 & 10 & 13 & 11 & 53 \\
\hline Incidence density ${ }^{a}$ & 39.1 & 16.2 & 19.8 & 18.2 & 22.4 \\
\hline aHR & 2.647 & 0.951 & 1.388 & Reference & - \\
\hline$(95 \% \mathrm{Cl})^{\mathrm{b}}$ & (1.148-6.103) & $(0.350-2.585)$ & $(0.580-3.322)$ & & \\
\hline \multicolumn{6}{|l|}{ Cancer } \\
\hline Number of deaths & 55 & 60 & 50 & 48 & 213 \\
\hline Incidence densitya & 113.2 & 97.3 & 76.2 & 79.5 & 90.1 \\
\hline aHR & 1.604 & 1.564 & 1.191 & Reference & - \\
\hline$(95 \% \mathrm{Cl})^{\mathrm{b}}$ & $(1.024-2.513)$ & $(1.020-2.398)$ & $(0.762-1.860)$ & & \\
\hline \multicolumn{6}{|l|}{ Other causes } \\
\hline Number of deaths & 33 & 28 & 35 & 36 & 132 \\
\hline Incidence density ${ }^{a}$ & 67.9 & 45.4 & 53.3 & 59.6 & 55.9 \\
\hline $\mathrm{aHR}$ & 1.013 & 0.658 & 0.894 & reference & - \\
\hline$(95 \% \mathrm{Cl})^{\mathrm{b}}$ & $(0.601-1.706)$ & $(0.381-1.134)$ & $(0.543-1.472)$ & & \\
\hline
\end{tabular}

Abbreviations: aHR, adjusted hazard ratio; $\mathrm{Cl}$, confidence intervals.

aper 100000 person-year.

baHR $(95 \% \mathrm{Cl})$ by the Cox proportional hazard model adjusted for age, sex, history of diabetes and/or hypertension medication, smoking, regular exercise, regular alcohol ingestion, body mass index, systolic blood pressure, fasting serum glucose, serum uric acid, serum albumin, glomerular filtration rate estimated with the Chronic Kidney Disease Epidemiology Collaboration creatinine equation, serum triglyceride, serum high-density lipoprotein-cholesterol and albuminuria.

Table 3 Association between serum bicarbonate level and all-cause mortality stratified by sex, body mass index and serum uric acid level

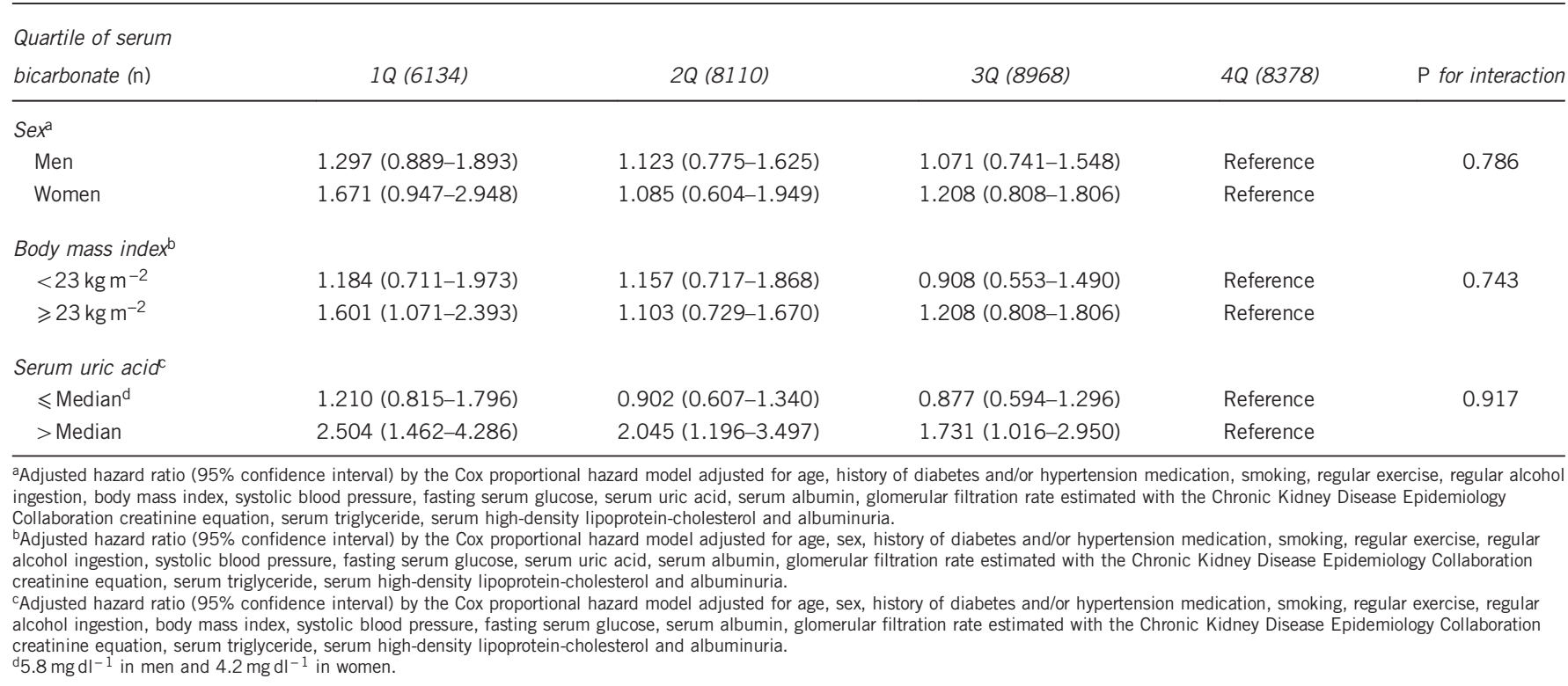

level of serum uric acid, lower serum bicarbonate was associated with mortality only in higher uric acid group although $\mathrm{p}$ for interaction was not significant (Table 3).

Acidic and neutral urine $\mathrm{pH}$ was associated with higher all-cause mortality compared to alkaline urine $\mathrm{pH}$ (aHR 2.550, 95\% CI 1.316-4.935, aHR 2.376, 95\% CI 1.254-4.501, respectively; Figure 1). Urine $\mathrm{pH}$ also tended to be associated with increased cardiovascular and cancer mortality, but the association was not statistically significant (data not shown). The association between the quartile groups of serum anion gap and all-cause mortality showed a similar trend but was not significant (Figure 1). When the serum anion gap was adjusted for serum albumin, the association was not significant either.

\section{DISCUSSION}

This study observed that clinical biomarkers of a higher metabolic acid load, such as lower serum bicarbonate level and urine $\mathrm{pH}$, were 


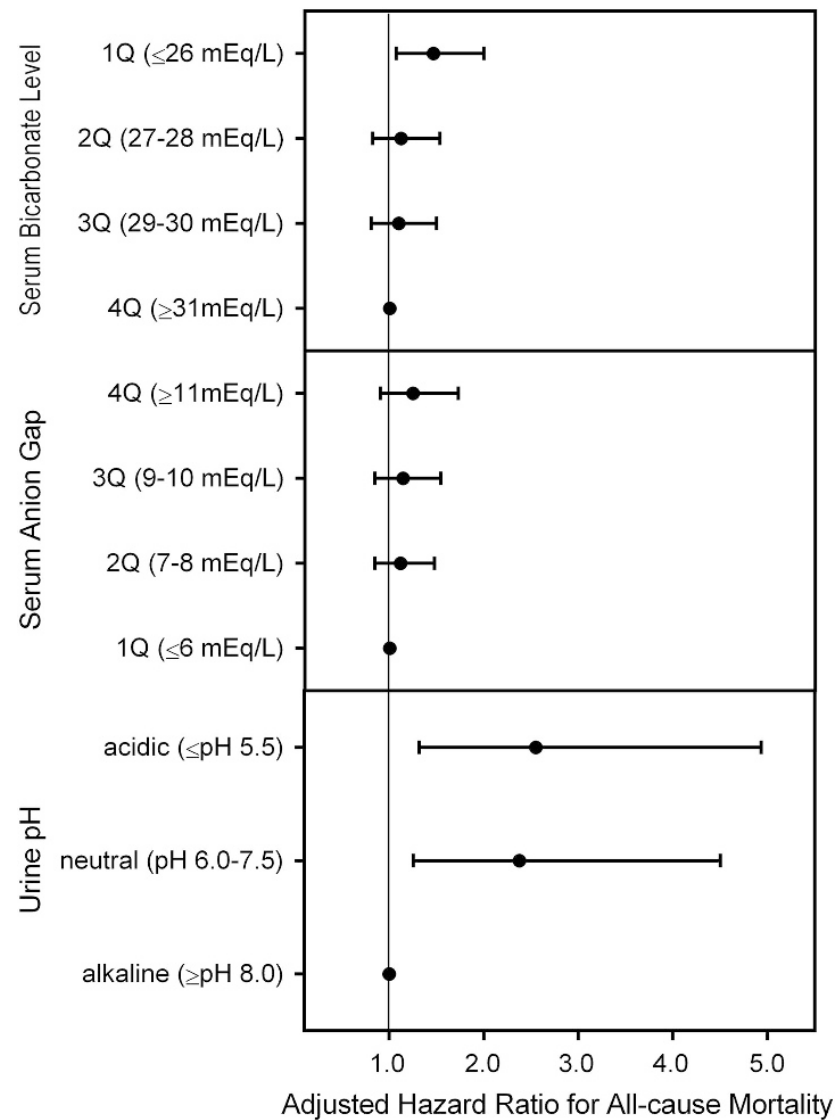

Figure 1 The association between clinical biomarkers of metabolic acid load and all-cause mortality. Adjusted hazard ratio based on the Cox proportional hazard model adjusted for baseline variables such as age, sex, history of diabetes and/or hypertension medication, smoking status, regular exercise, regular alcohol ingestion, body mass index, systolic blood pressure, serum calcium, serum phosphorus, serum uric acid, serum albumin, fasting serum glucose, serum high-density lipoprotein-cholesterol, serum triglyceride, albuminuria and estimated glomerular filtration rate calculated with the Chronic Kidney Disease Epidemiology Collaboration creatinine equation. Error bars represent the $95 \%$ confidence interval.

associated with a higher all-cause mortality in an apparently healthy population after a median follow-up of 93 months. Higher serum anion gap tended to be associated with higher all-cause mortality, but the association was not significant. The association between lower serum bicarbonate level and higher all-cause mortality was more prominent in participants with BMI higher than $23 \mathrm{~kg} \mathrm{~m}^{-2}$, although the $P$-value for the interaction did not reach statistical significance.

These findings are consistent with previous studies regarding the association between metabolic acid load and mortality risk factors, such as insulin resistance, ${ }^{2}$ inflammatory biomarkers, ${ }^{3}$ visceral obesity and metabolic syndrome, ${ }^{4}$ high $\mathrm{BP}^{5,6}$ and low cardiorespiratory fitness ${ }^{7}$ in the general population. Evidence of an association between increased metabolic acid load and increased mortality in the general population with preserved renal function is very limited. Raphael et al. ${ }^{12}$ reported no association between serum bicarbonate and allcause mortality in adults without CKD after a mean follow-up of 8.7 years. In adults without $\mathrm{CKD}$, the aHR of subjects with a serum bicarbonate level less than $22 \mathrm{~mm}$ for all-cause mortality was 1.56 (95\% CI 0.78-3.09) compared with those with a serum bicarbonate level of $31 \mathrm{~mm}$ or higher. Among the 15836 adults in that study, the proportion of those without CKD was $92 \%$ and their mean age was
43.4 years. Therefore, the possibility of a lack of statistical power because of the relatively small sample size and short follow-up period considering the rather young study population cannot be excluded. Ahn et al. ${ }^{19}$ reported that a higher serum anion gap was predictive of mortality in the elderly in a community-based cohort in Korea. In that study, the eGFR range of participants was $5.7-129.0 \mathrm{ml} \mathrm{min}^{-1}$ per $1.73 \mathrm{~m}^{2}$ and participants with an impaired renal function were included. Therefore, it was not possible to differentiate the risk of mortality associated with CKD from that associated with an increased metabolic acid load itself.

The bicarbonate level of the first quartile was within the normal range and that of the fourth quartile was beyond the upper normal limit. The mortality risk was, however, highest in the first quartile and lowest in the fourth quartile. This observation may bring forward the necessity of reconsidering the normal range of serum bicarbonate level.

The present study showed a significant association between the lower serum bicarbonate or acidic urine $\mathrm{pH}$ and all-cause mortality in a healthy population with a preserved renal function. The explanation for the association between metabolic acid load and mortality is not clear. In this study, the association between serum bicarbonate and mortality was more prominent in participants with higher BMI $\left(>23 \mathrm{~kg} \mathrm{~m}^{-2}\right)$ and serum uric acid level higher than the sex-specific median (5.8 $\mathrm{mg} \mathrm{dl}^{-1}$ in men and $4.2 \mathrm{mg} \mathrm{dl}^{-1}$ in women) at baseline, although the $P$-value for the interaction was not significant. Therefore, it is possible that obesity plays a pathophysiological role in the association between metabolic acid load and mortality. Obesity is a well-known risk factor for all-cause, cardiovascular and cancer morality. ${ }^{20}$ The association between the biomarkers of metabolic acid load and an increased cardiovascular mortality is consistent with the previous observational studies confirmed the association between diet-induced metabolic acidosis and insulin resistance and systemic hypertension, and therefore the risk of cardiovascular diseases. ${ }^{21}$ Diet-induced acidosis is a well-known risk factor for cancer, and the association is explained by adrenal glucocorticoid, insulin-like growth factor and adipocyte cytokine signaling, dysregulated cellular metabolism and osteoclast activation. A possible effect of obesity on the link between acidogenic diet and cancer risk has been suggested. ${ }^{22}$ The role of obesity and fat distribution in the association between metabolic acid load and mortality deserves further researches in the future. The role of serum uric acid level in the association between the lower serum bicarbonate and mortality needs to be confirmed.

Compared with serum bicarbonate and urine $\mathrm{pH}$, serum anion gap was not significantly associated with mortality. After adjustment of serum anion gap with serum albumin, the association was still not significant. The explanation for this lack of association is not clear and further research is necessary. COPD can influence the serum bicarbonate level. When we excluded participants who were diagnosed with COPD within 12 months after the health screening, the level of association did not change. Therefore, the influence of respiratory acidosis because of COPD on the correlation between serum bicarbonate and mortality could be reasonably excluded.

The present study was not able to identify a possible gender effect in the association between metabolic acid load and mortality. An association between metabolic acid load and type 2 diabetes or hypertension has been observed in previous studies of which participants were only women., ${ }^{8,9}$ Higher dietary acid load itself has been reported to be associated with higher systolic and diastolic BP in a cross-sectional study of women ${ }^{23}$ and with incident hypertension in a prospective study of women. ${ }^{24}$ Higher dietary acid load has also been 
associated with an increased risk of incident diabetes in a prospective study of women, ${ }^{25}$ but not in a prospective study of men. ${ }^{26}$ Although Engberink et al..$^{27}$ reported a negative association between dietary acid load and the risk of incident hypertension in 2241 participants, $43 \%$ of whom were men, they did not analyze the gender effect. Additional studies on larger populations with longer follow-up duration are necessary to confirm the possible gender effect on the association between metabolic acid load and mortality.

The present study is not without limitations. First, blood gas analysis including $\mathrm{CO}_{2}$ in blood was not available. Although respiratory acidosis can increase the serum bicarbonate level, after exclusion of participants who were diagnosed with COPD within 12 months of the health screening in this study, the level of the association between clinical biomarkers of metabolic acidosis such as lower serum bicarbonate and acidic urine $\mathrm{pH}$ and mortality did not change. Second, urine $\mathrm{pH}$ was measured with a urine dipstick. Although urine $\mathrm{pH}$ was not measured with a $\mathrm{pH}$ meter, the $\mathrm{pH}$ value measured with a urine dipstick was associated with mortality. It may be more clinically relevant because a $\mathrm{pH}$ meter is not always available in clinical settings. Third, clinical biomarkers of metabolic acid load were measured only once, and the chronicity of higher metabolic acid load was not clearly proved in this study. Because participants visited the hospital for a health screening, not for symptoms of illness, it is reasonable to assume that the metabolic acid load measured in the participants represented their steady-state acid-base balance. Fourth, information on diet was not available and the cause of higher metabolic acid load could not be analyzed. With detailed information on diet, it should have been possible to find plausible explanations for increased metabolic acid load in participants. Dietary behavior has been associated with various cardiovascular risk factors ${ }^{28}$ and dietary load of metabolic acid may be one of the mechanisms linking the dietary behavior and cardiovascular risk factors. Lastly, the results were derived from a retrospective study performed at a single center without structured protocols and on a single ethnicity. Therefore, generalization of the results should be cautious.

Despite these limitations, to the best of our knowledge, the present study is the first study showing an independent association between metabolic acid load and all-cause mortality in the general population with preserved renal function. Because of the rather long follow-up duration of a relatively large population, this study could analyze the association between metabolic acid load and all-cause and causespecific mortality.

In conclusion, a higher metabolic acid load was associated with an increased all-cause and cardiovascular mortality in adults with a preserved renal function. Additional studies are necessary to confirm the association between metabolic acid load and mortality and the causality of the relationship.

\section{CONFLICT OF INTEREST}

The authors declare no conflict of interest.

\section{ACKNOWLEDGEMENTS}

This work was supported by a grant from the National Research Foundation of Korea (NRF) funded by the Korean government (Ministry of Education and Science Technology) (2010-0028631)

Author contributions: MP and HJY designed the study, analyzed and interpreted the data and prepared the manuscript. SJJ and SY collected and analyzed the data. JMY designed the study and collected the data. All authors reviewed and edited the manuscript and approved the final version of the manuscript.
1 Scialla JJ, Anderson CA. Dietary acid load: a novel nutritional target in chronic kidney disease? Adv Chronic Kidney Dis 2013; 20: 141-149.

2 Farwell WR, Taylor EN. Serum bicarbonate, anion gap and insulin resistance in the National Health and Nutrition Examination Survey. Diabet Med 2008; 25: 798-804.

3 Farwell WR, Taylor EN. Serum anion gap, bicarbonate and biomarkers of inflammation in healthy individuals in a national survey. CMAJ 2010; 182: 137-141.

4 Otsuki M, Kitamura T, Goya K, Saito H, Mukai M, Kasayama S, Shimomura I, Koga M. Association of urine acidification with visceral obesity and the metabolic syndrome. Endocr J 2011; 58: 363-367.

5 Taylor EN, Forman JP, Farwell WR. Serum anion gap and blood pressure in the National Health and Nutrition Examination Survey. Hypertension 2007; 50: 320-324.

6 Forman JP, Rifas-Shiman SL, Taylor EN, Lane K, Gillman MW. Association between the serum anion gap and blood pressure among patients at Harvard Vanguard Medical Associates. J Hum Hypertens 2008; 22: 122-125.

7 Abramowitz MK, Hostetter TH, Melamed ML. Lower serum bicarbonate and a higher anion gap are associated with lower cardiorespiratory fitness in young adults. Kidney Int 2012; 81: 1033-1042.

8 Mandel EI, Curhan GC, Hu FB, Taylor EN. Plasma bicarbonate and risk of type 2 diabetes mellitus. CMAJ 2012; 184: E719-E725.

9 Mandel EI, Forman JP, Curhan GC, Taylor EN. Plasma bicarbonate and odds of incident hypertension. Am J Hypertens 2013; 26: 1405-1412.

10 Driver TH, Shlipak MG, Katz R, Goldenstein L, Sarnak MJ, Hoofnagle AN, Siscovick DS Kestenbaum B, de Boer IH, Ix JH. Low serum bicarbonate and kidney function decline: the Multi-Ethnic Study of Atherosclerosis (MESA). Am J Kidney Dis 2014; 64 534-541.

11 Goldenstein L, Driver TH, Fried LF, Rifkin DE, Patel KV, Yenchek RH, Harris TB Kritchenvsky SB, Newman AB, Sarnak MJ, Shlipak MG, Ix JH. Health ABC Study Investigators. Serum bicarbonate concentrations and kidney disease progression in community-living elders: The Health, Aging, and Body Composition (Health ABC) Study. Am J Kidney Dis 2014; 64: 542-549.

12 Raphael KL, Zhang Y, Wei G, Greene T, Cheung AK, Beddhu S. Serum bicarbonate and mortality in adults in NHANES III. Nephrol Dial Transplant 2013; 28: 1207-1213.

13 Frassetto L, Morris RC Jr, Sellmeyer DE, Todd K, Sebastian A. Diet, evolution and aging -the pathophysiologic effects of the post-agricultural inversion of the potassium-tosodium and base-to-chloride ratio in the human diet. Eur J Nutr 2001; 40: 200-213.

14 Cordain L, Eaton SB, Sebastian A, Mann N, Lindeberg S, Watkins BA, O'Keefe JH, Brand-Miller J. Origins and evolution of the Western diet: health implications for the 21st century. Am J Clin Nutr 2005; 81: 341-354.

15 Mastushita K, Mahmoodi BK, Woodward M, Emberson JR, Jafar TH, Jee SH, Polkinghorne KR, Shankar A, Smith DH, Tonelli M, Warnock DG, Wen CP, Coresh J, Ganevoort RT, Hemmelgarn BR, Levey AS. Chronic Kidney Disease Prognosis Consortium. Comparison of risk prediction using the CKD-EPI equation and the MDRD study equation for estimated glomerular filtration rate. JAMA 2012; 307: 1941-1951.

16 Inker LA, Schmid CH, Tiqhiouart H, Eckfeldt JH, Feldman HI, Greene T, Kusek JW, Manzi J, Van Lente F, Zhang YL, Coresh J, Levey AS. CKD-EPI Investigators. Estimating glomerular filtration rate from serum creatinine and cystatin C. N Engl J Med 2012; 367: 20-29.

17 Kraut JA, Madias NE. Metabolic acidosis: pathophysiology, diagnosis and management. Nat Rev Nephrol 2010; 6: 274-285.

18 World Health Organization; International Obesity Task Force. The Asian-Pacific Perspective: Redefining Obesity and Its Treatment. WHO Western Pacific Region: Geneva, Switzerland, 2000.

19 Ahn SY, Ryu J, Baek SH, Han JW, Lee JH, Ahn S, Kim KI, Chin HJ, Na KY, Chae DW, Kim KW, Kim S. Serum anion gap is predictive of mortality in an elderly population. Exp Gerontol 2014; 50: 122-127.

20 Lenz M, Richter T, Mühlhauser I. The morbidity and mortality associated with overweight and obesity in adulthood: a systematic review. Ditsch Arztebl Int 2009; 106: 641-648.

21 Adeva MM, Souto G. Diet-induced metabolic acidosis. Clin Nutr 2011; 30: 416-421.

22 Robey IF. Examining the relationship between diet-induced acidosis and cancer. Nutr Metab 2012; 9: 72.

23 Murakami K, Sasaki S, Takahashi Y, Uenishi K. Japan Dietetic Students' Study for Nutrition and Biomarkers Group. Association between dietary acid-base load and cardiometabolic risk factors in young Japanese women. $\mathrm{Br} J$ Nutr 2008; 100 642-651.

24 Zhang L, Curhan GC, Forman JP. Diet-dependent net acid load and risk of incident hypertension in United States women. Hypertension 2009; 54: 751-755.

25 Fagherazzi G, Vilier A, Bonnet F, Lajous M, Balkau B, Boutron-Rualt MC Clavel-Chapelon F. Dietary acid load and risk of type 2 diabetes: the E3N-EPIC cohort study. Diabetologia 2014; 57: 313-320.

26 Xu H, Jia T, Huang X, Risérus U, Cederholm T, Arnlöv J, Sjögren P, Lindholm B, Carrero JJ. Dietary acid load, insulin sensitivity and risk of type 2 diabetes in community-dwelling older men. Diabetologia 2014; 57: 1561-1568.

27 Engberink MF, Bakker SJ, Brink EJ, van Baak MA, van Rooij FJ, Hofman A, Witteman JC, Geleijnse JM. Dietary acid load and risk of hypertension: the Rotterdam Study. Am J Clin Nutr 2012; 95: 1438-1444.

28 Umesawa M, Kitamura A, Kiyama M, Okada T, Shimizu Y, Imano H, Ohira T, Nakamura M, Maruyama K, Iso H. CIRCS Investigators. Association between dietary behavior and risk of hypertension among Japanese male workers. Hypertens Res 2013; 36: $374-380$ 